\title{
Performance Monitoring for Sensorimotor Confidence: A Visuomotor Tracking Study
}

\author{
Shannon M. Locke ${ }^{1,2^{*}}$, Pascal Mamassian ${ }^{1}$, Michael S. Landy ${ }^{2,3}$ \\ 1 Laboratoire des Systèmes Perceptifs, Département d'Études Cognitives, École Normale \\ Supérieure, PSL University, CNRS, 75005 Paris, France \\ 2 Department of Psychology, New York University, New York, NY, United States \\ 3 Center for Neural Science, New York University, New York, NY, United States \\ * corresponding author: shannon.m.locke@nyu.edu
}

\section{Abstract}

To best interact with the external world, humans are often required to consider the quality 2 of their actions. Sometimes the environment furnishes rewards or punishments to signal 3 action efficacy. However, when such feedback is absent or only partial, we must rely on 4 internally generated signals to evaluate our performance (i.e., metacognition). Yet, very 5 little is known about how humans form such judgements of sensorimotor confidence. Do 6 they monitor their performance? Or do they rely on cues to sensorimotor uncertainty to 7 infer how likely it is they performed well? We investigated motor metacognition in two 8 visuomotor tracking experiments, where participants followed an unpredictably moving dot 9 cloud with a mouse cursor as it followed a random trajectory. Their goal was to infer the 10 underlying target generating the dots, track it for several seconds, and then report their ${ }_{11}$ confidence in their tracking as better or worse than their average. In Experiment 1, we ma- 12 nipulated task difficulty with two methods: varying the size of the dot cloud and varying 13 the stability of the target's velocity. In Experiment 2, the stimulus statistics were fixed and 14 duration of the stimulus presentation was varied. We found similar levels of metacognitive 15 sensitivity in all experiments, with the temporal analysis revealing a recency effect, where 16 error later in the trial had a greater influence on the sensorimotor confidence. In sum, these ${ }_{17}$ results indicate humans predominantly monitor their tracking performance, albeit ineffi- ${ }_{18}$ ciently, to judge sensorimotor confidence. 
Keywords: sensorimotor, confidence, metacognition, perception, action, tracking.

\section{Highlights}

- Participants consciously reflected on their tracking performance with some accuracy ${ }_{23}$

- Sensorimotor confidence was influenced by recent errors

- Expectations of task difficulty did not play a large role in sensorimotor confidence

- Metacognitive sensitivity of binary confidence judgements on continuous performance 26 can be quantified with standard non-parametric techniques

\section{Introduction}

Sensorimotor decision-making is fundamental for humans and animals when interacting 29 with their environment. For example, it will determine where we look, how we move our 30 limbs through space, or what actions we select to intercept or avoid objects. In return, 31 we may receive decision feedback from the environment, such as resources, knowledge, 32 social standing, injury, or embarrassment. Such outcomes of an action are often crucial for ${ }_{3}$ determining subsequent sensorimotor decision-making, particularly in dynamic scenarios 34 where a series of actions are chained together to achieve a sensorimotor goal (e.g., dancing or 35 tracking a target). But what happens if external feedback is absent, partial, or significantly ${ }_{36}$ delayed? How then do we judge if an action has been performed well? One possible solution $\quad 37$ is for the action taker to form their own subjective evaluation of sensorimotor performance 38 using whatever sensory or motor signals are available. These metacognitive judgements 39 reflect the person's confidence that their action or series of actions accomplished their 40 sensorimotor goal. Yet, despite such judgements being a familiar and everyday occurrence, ${ }_{41}$ 
they have received relatively little direct scientific scrutiny.

Different related elements of sensorimotor confidence have been touched upon in a vari- ${ }_{43}$ ety of domains, highlighting the sophisticated monitoring and control processes of the brain ${ }_{44}$ that operate on internally-gathered information (see Figure 1 for a summary). At a broader 45 level, there is the topic cognitive control, which describes how goals or plans translate into 46 actual behaviour. It is thought that cognitive control, also known as executive control, ${ }_{47}$ is responsible for the appropriate deployment of attention, as well as voluntary selection, 48 initiation, switching, or termination of tasks (Norman and Shallice, 1986; Botvinick et al., 49 2001; Alexander and Brown, 2010). At a finer level is the study of sensorimotor control. 50 Usually, research questions focus on how the brain senses discrepancies between the in- ${ }_{51}$ tended outcome of motor commands, as specified by an internal model, and the actual ${ }_{52}$ action outcomes, that are processed as a feedback signal, to correct and update subsequent 53 motor control signals (Wolpert et al., 1995; Todorov, 2004). While the understanding of 54 sensorimotor processes is quite advanced, both at the behavioural and neural levels, very ${ }_{55}$ little is known about our ability to consciously monitor sensorimotor performance. 56

If the action is reduced to a simple report of what is perceived, the monitoring of senso- ${ }_{57}$ rimotor performance reduces to the study of perceptual confidence (Pleskac and Busemeyer, $\quad 58$ 2010; Fleming and Dolan, 2012; Mamassian, 2016). Perceptual confidence is a metacogni- 59 tive process, which corresponds to the subjective sense of the correctness of our perceptual 60 decisions (Galvin et al., 2003; Pouget et al., 2016). Human observers exhibit considerable 61 sensitivity to the quality of the processing of sensory information and the resulting abil- ${ }_{62}$ ity to predict the correctness of the perceptual choice (Barthelmé and Mamassian, 2010; 63 Kiani et al., 2014; Adler and Ma, 2018). However this so-called Type-2 judgement often 64 incurs additional noise, on top of the sensory noise that reduces perceptual performance 65 (Type-1 decisions) (Maniscalco and Lau, 2016). More recently, researchers have considered 66 the contribution of motor factors in perceptual confidence (Yeung and Summerfield, 2012; 67 Kiani et al., 2014; Fleming and Daw, 2017). Such elements are crucial, for example, for ${ }_{68}$ 


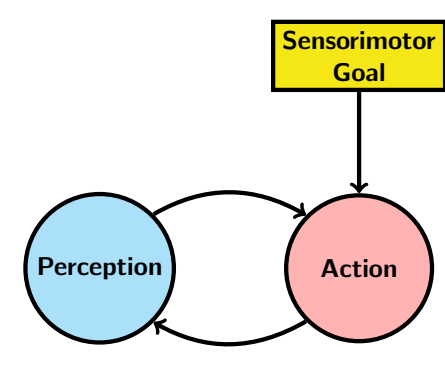

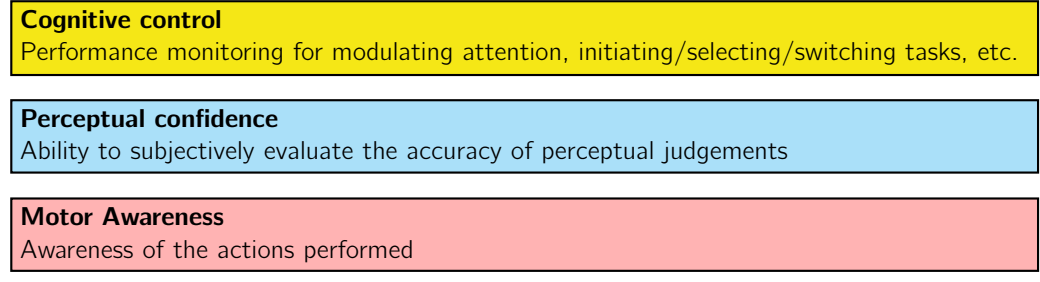

Uncertainty cues to difficulty (prospective)

Knowledge of perceptual uncertainty and/or motor noise that predicts performance

Feedback cues to performance (retrospective)

Evaluation of sensorimotor error signals from internal models, proprioception, vision, etc.

Figure 1: Components of sensorimotor control (left) and related topics in the literature (right). Sensorimotor confidence is a subjective evaluation of how well behaviour fulfilled the sensorimotor goal, considering both sensory and motor factors. The topic of sensorimotor confidence is complementary to the discussions of cognitive control, perceptual confidence, motor awareness, uncertainty, and self-generated feedback. It is likely that cues to difficulty and performance, that are responsible for the computation of sensorimotor confidence, originate both from sensory and motor sources. The former cues are prospective as they are related to how well the acting agent can potentially perform, whereas the latter are retrospective, they become available only after the action has occurred.

the observer to respond "low confidence" on lapse trials where they mistakenly pressed the 69 wrong key. In other examples, motor behaviour is used as an index of perceptual confidence by tracking hand kinematics while observers report their perceptual judgement (Resulaj $\quad{ }_{71}$ et al., 2009; Patel et al., 2012; Dotan et al., 2018). However, these noted contributions are 72 often restricted to simple motor behaviours, and do not take into account motor sources of 73 variability.

Motor awareness, the degree to which we are conscious of the actions we take (Blake- ${ }_{75}$ more et al., 2002; Blakemore and Frith, 2003), is also likely to contribute to sensorimotor 76 confidence. Not all actions are consciously monitored, and it is a common experience to act 77 without conscious control. For example, when we are walking, we are not always thinking 78 of exactly how to place one foot in front of the other. Yet, for other actions, we must 79 consciously attend to them, such as threading a sewing needle. A seminal study on motor 80 awareness by Fourneret and Jeannerod (1998) found poor introspective ability for the ac- $\quad 81$ tion made when an unseen hand movement is perturbed by a horizontal displacement in 82 
the visual feedback signal. Participants discount their compensatory actions and instead 83 indicated that their hand position followed a trajectory much like the perturbed cursor. $\quad{ }^{84}$ Follow-up studies have modified the response to be a binary motor-awareness decision (e.g., $\quad 85$ "Was feedback perturbed or not") followed by a confidence rating (Sinanaj et al., 2015; 86 Bègue et al., 2018). Another motor-awareness study measured confidence ratings following $\quad 87$ a judgement of whether a visual dot was flashed ahead or behind their finger position dur- 88 ing up-down movement (Charles et al., 2020). However, we shall argue that none of these 89 measurements of confidence correspond to sensorimotor confidence as we have defined it. 90 Motor-awareness confidence reflects the knowledge held about the executed actions, whereas 91 we propose that sensorimotor confidence involves an additional step of evaluating how well 92 these behaviours serve the sensorimotor goal. For example, a competitive swimmer might ${ }_{93}$ know all the moves they executed to reach the other end of the pool (i.e., motor aware- ${ }_{94}$ ness), and confidently report said awareness, but they might find it difficult to judge if they 95 completed this lap faster than usual (i.e., sensorimotor confidence). To our knowledge, the 96 only study to ask participants to explicitly reflect on their sensorimotor performance was 97 by Mole et al. (2018), who had participants perform a virtual driving task. Green lines 98 were placed on the road to indicate a good-performance zone, and after completing the 99 trial, they were asked to report the percentage of time they spent in the green zone (i.e., a 100 continuous measure of sensorimotor confidence). They found that correspondence between 101 objective performance and sensorimotor confidence roughly followed difficulty of the task 102 $\begin{array}{ll}\text { but was otherwise limited. } & 103\end{array}$

The study of sensorimotor confidence should be contrasted with the mere knowledge of 104 sensorimotor uncertainty (Augustyn and Rosenbaum, 2005). In theory, this can be studied 105 by examining how knowledge of variability from sensory, motor, and task sources, influ- 106 ences motor decision-making (Wolpert and Landy, 2012). The majority of studies support 107 the hypothesis that humans plan actions consistent with accurate knowledge of their sen- 108 sorimotor uncertainty (e.g., Augustyn and Rosenbaum, 2005; Trommershäuser et al., 2008; 109 
Stevenson et al., 2009; Bonnen et al., 2015), with some exceptions (e.g., Zhang et al., 2013). 110 However, the degree to which this knowledge is consciously available to the person is highly 111 debatable (Augustyn and Rosenbaum, 2005). Furthermore, judgements of one's uncertainty 112 in a planned action only allow one to predict the probability of a successful outcome. In 113 this sense, they can act as prospective confidence judgements before the action is taken, 114 but do not constitute retrospective confidence judgements made by reflecting on sensorimo- 115 tor behaviour from performance monitoring. For example, one would typically have more 116 prospective confidence for riding a bicycle than a unicycle. This belief is not derived from 117 performance monitoring but rather from experience-informed expectation. In other areas 118 of metacognitive research, such use of uncertainty information or other predictions of task 119 difficulty are considered heuristics that can even impair the relationship between objective 120 performance and confidence (e.g., Spence et al., 2015; De Gardelle and Mamassian, 2015; 121 Mole et al., 2018; Charles et al., 2020). Thus, it is desirable to identify the degree to which 122 sensorimotor confidence is based on conscious monitoring of performance from feedback ${ }_{123}$ cues versus prospective judgements of performance based on uncertainty cues. 124

Here, we report on two experiments explicitly measuring sensorimotor confidence in a 125 visuomotor tracking task. In both experiments, participants manually tracked a target, 126 the location of which was inferred from limited noisy sensory information, a twinkling dot 127 cloud, as it followed an unpredictable trajectory. Afterwards, they reported their sensori- ${ }_{128}$ motor confidence by subjectively evaluating their performance with a relative judgement of ${ }_{129}$ "better" or "worse" than their average. A dynamic task was selected to mirror the sensori- 130 motor goals typically encountered in the real world. In Experiment 1, we manipulated the 131 difficulty of the task by changing the uncertainty of the location of the sensory stimulus 132 with two separate methods. In Experiment 2, we manipulated the stimulus-presentation 133 duration to introduce uncertainty about when the confidence response would be required. $\quad 134$ We had several goals in this study: 1) to test whether humans are able to make reasonable ${ }_{135}$ sensorimotor confidence judgements from monitoring performance error signals rather than 136 
relying only on uncertainty-based expectations; 2) to quantify how well sensorimotor confi- ${ }_{137}$ dence reflected objective performance; and 3) to examine what evidence contributed to the ${ }_{138}$ sensorimotor confidence judgement.

\section{Experiment 1}

Experiment 1 sought to measure sensorimotor confidence in a visuomotor tracking task ${ }_{141}$ and establish a metric of metacognitive sensitivity that quantified how well the confidence 142 judgements corresponded to objective tracking performance. Difficulty in the task was ${ }_{143}$ manipulated in the cloud-size session by varying the external noise of the sensory evidence 144 indicating the target location. In the velocity-stability session, we varied the degree of noise ${ }_{145}$ in the target's trajectory. To investigate the error evidence contributing to the sensorimotor ${ }_{146}$ confidence, we investigated the temporal pattern of metacognitive sensitivity, applying our ${ }_{147}$ metric to $1 \mathrm{~s}$ time bins within the trial.

\subsection{Methods}

Participants: Seven naive participants (23 - 35 years old, one left-handed, one female) ${ }_{150}$ took part in the study. All had normal or corrected-to-normal vision and self-reported 151 normal motor functioning. They received details of the experimental procedures and gave 152 informed consent prior to the experiment. Participants were tested in accordance with the ${ }_{153}$ ethics requirements of the École Normale Supérieure and the Declaration of Helsinki. ${ }_{154}$

Apparatus: Stimuli were displayed on a V3D245 LCD monitor (Viewsonic, Brea, CA; 155 $52 \times 29.5 \mathrm{~cm}, 1920 \times 1080$ pixels, $60 \mathrm{~Hz}$ ). Participants sat $46.5 \mathrm{~cm}$ from the monitor with 156 their head stabilised by a chin rest. Manual tracking was performed using a Logitech M325 157 wireless optical mouse, operated by the participant's right hand. Subjective assessments 158 of performance were reported on a standard computer keyboard with the left hand. The ${ }_{159}$ experiment was conducted using custom-written code in MATLAB version R2014a (The 160 
MathWorks, Natick, MA), using Psychtoolbox version 3.0.12 (Brainard, 1997; Pelli, 1997; ${ }_{161}$ Kleiner et al., 2007).

Dot-cloud stimulus: Every frame, two white dots were drawn from a 2D circularly 163 symmetric Gaussian generating distribution with standard deviation $\sigma_{\text {cloud }}$. The mean of the 164 distribution was the tracking target, which was invisible to observers and must be inferred 165 from the dot cloud. Each dot had a one-frame lifetime and new dots were drawn every 166 frame. Due to the persistence of vision, participants had the impression of seeing up to $10 \quad 167$ dots at any one time (Figure 2A). Dots had a diameter of $0.25 \mathrm{deg}$ and were presented on 168 a mid-grey background. Dots were generated using Psychtoolbox functions that rendered 169 them with sub-pixel dot placement and high quality anti-aliasing. The horizontal position 170 of the target changed every frame according to random walk in velocity space (Figure 2B): 171 $v_{t+1}=v_{t}+\epsilon$ and $\epsilon \sim \mathcal{N}\left(0, \sigma_{\text {walk }}\right) \mathrm{deg} / \mathrm{s}$. This gave the target momentum, making it more ${ }_{172}$ akin to a real-world moving target (Figure 2C). Both the target and the black cursor dot 173 (diam.: $0.19 \mathrm{deg}$ ) were always centred vertically on the screen. Trajectories that caused 174 the target to move closer than $2 \times \max \left(\sigma_{\text {cloud }}\right)$ from the screen edge were discarded and 175 resampled prior to presentation.

Task: The trial sequence (Figure 2D) began with a red dot at the centre of the screen. ${ }_{177}$ Participants initiated the tracking portion of the trial by moving the black cursor dot to this 178 red dot, causing the red dot to disappear. The dot-cloud stimulus appeared immediately, 179 with the target centred horizontally. The target followed its horizontal random walk for 180 10 s. Then, the participant made a subjective assessment of tracking performance while 181 viewing a blank grey screen, reporting by keypress whether they believed their tracking 182 performance was better or worse than their session average. The experiment was conducted 183 in two sessions on separate days. In the "cloud size" session, the standard deviation of the 184 dot cloud, $\sigma_{\text {cloud, }}$, was varied from trial to trial (5 levels: 1, 1.5, 2, 2.5, and 3 deg) and 185

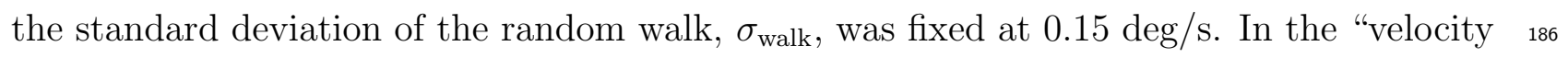
stability" session, $\sigma_{\text {walk }}$ was varied (5 levels: $0.05,0.10,0.15,0.20$, and $0.25 \mathrm{deg} / \mathrm{s}$ ) and $\sigma_{\text {cloud }} 187$ 
A

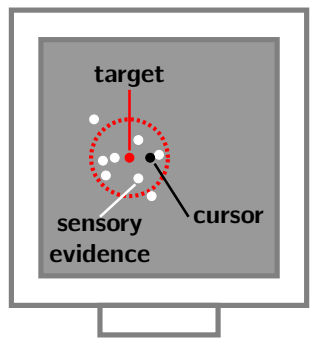

B

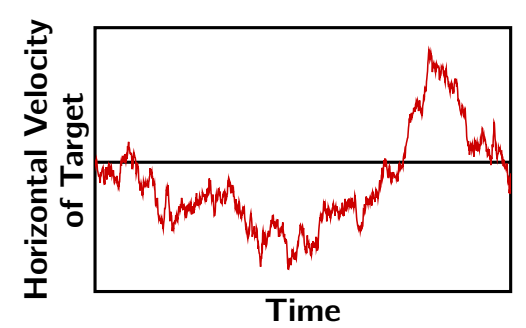

C

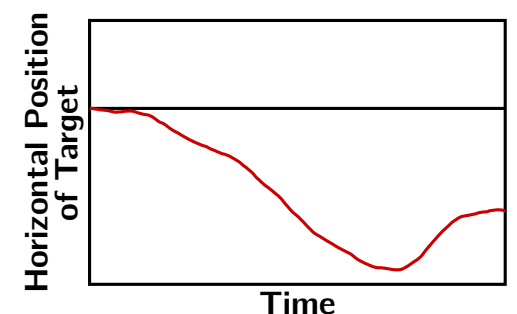

D

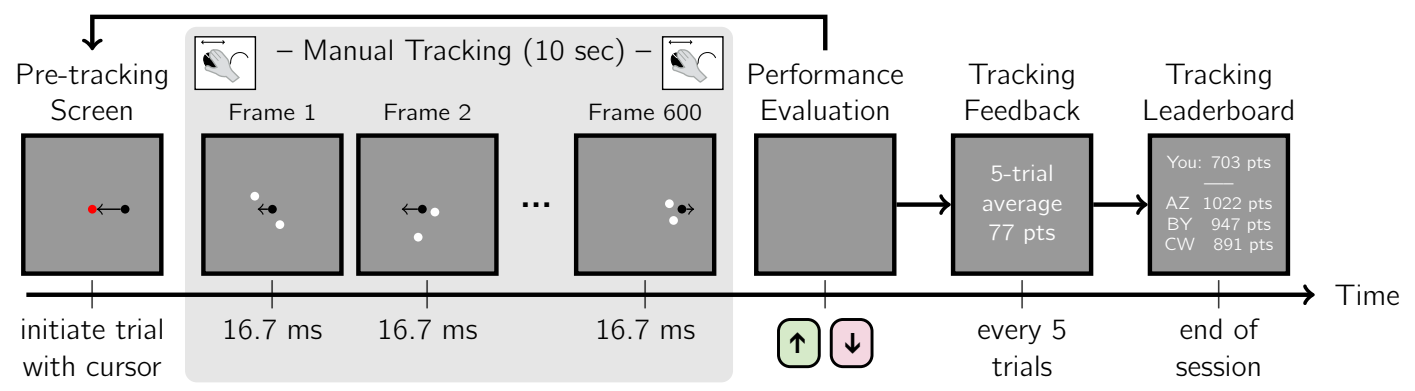

Figure 2: Visuomotor tracking task. A: The "twinkling" dot cloud stimulus (white), generated by drawing two dots per frame from a 2D Gaussian generating distribution. Red: mean and 1 SD circle, which were not displayed. Black: mouse cursor. The dots provided sensory evidence of target location (generating distribution mean). As shown, more than two dots were perceived at any moment due to temporal averaging in the visual system. B: Example target random-walk trajectory in velocity space. C: The corresponding horizontal trajectory of the target. D: Trial sequence. Trials were initiated by the observer, followed by $10 \mathrm{~s}$ of manual tracking of the inferred target with a computer mouse. Then, participants reported their sensorimotor confidence by indicating whether their performance on that trial was better or worse than their average. Objective performance feedback was provided intermittently including average points awarded and a final leaderboard. Difficulty manipulations: cloud size $\left(\sigma_{\text {cloud }}\right)$ and velocity stability $\left(\sigma_{\text {walk }}\right)$ were varied in separate sessions. 
was fixed at 2 deg. Examples of the stimuli for both sessions are provided as Supplementary $\quad{ }_{188}$ media files. The order of sessions was counterbalanced across participants to the best extent 189 possible. Each session began with a training block (20 trials, 4 per stimulus level in random 190 order), where only tracking responses were required. The training trials allowed participants 191 to become familiar with the stimulus and set-up, and to form an estimate of their average 192 performance. The main testing session followed (250 trials, 50 per stimulus level in random 193 order). For the second session, participants were instructed to form a new estimate of 194 average performance, and not to rely on their previous estimate.

Grading objective performance: For all analyses, we used root-mean-squared-error 196 (RMSE) as our measure of tracking error, calculated from the horizontal distance between 197 the target (i.e., the current distribution mean) and the cursor. For the purposes of feedback, 198 the tracking performance on each trial was converted to a score according to the formula 199 points $=100-30 * R M S E$. Typical scores ranged from 60 to 80 points. Every 5 trials, 200 the average score for the previous 5-trials was reported. This feedback was provided for 201 both training and test trials. Presenting the average score served several purposes. It 202 1) incentivised good tracking behaviour; 2) encouraged consistent performance across the 203 session; and 3) helped participants to maintain a calibrated internal estimate of average ${ }^{204}$ performance. At the end of a session, participants were shown their cumulative score for 205 that session and ranking on a performance leaderboard.

Metacognitive sensitivity metric: To examine sensorimotor confidence, we sought 207 a metacognitive sensitivity metric that reflected how well the confidence reports identified 208 good from bad tracking performance (i.e., low versus high RMSE). This concept is similar 209 to the one used in perceptual confidence, where metacognitive sensitivity refers to a per- ${ }_{210}$ son's ability to distinguish correct from incorrect decisions (Fleming and Lau, 2014). As the ${ }_{211}$ outcome of tracking was not binary (e.g., correct vs. incorrect), we considered the objective ${ }_{212}$ tracking performance within a trial relative to all trials within the session performed by ${ }^{213}$ that participant. We constructed two objective-performance probability distributions con- ${ }^{214}$ 
ditioned on the sensorimotor confidence: one distribution for trials followed by a "better ${ }_{215}$ than average" response and one for "worse than average" responses (Figure 3A-B). A high ${ }_{216}$ overlap in these conditional distributions would reflect low metacognitive sensitivity as this 217 means objective performance is a poor predictor of the participant's evaluation of their ${ }_{218}$ performance. Conversely, low overlap indicates high metacognitive sensitivity. We used an ${ }^{219}$ empirical Receiver Operating Characteristic (ROC) curve, also known as a quantile-quantile 220 plot (Figure 3C), for a non-parametric measure of metacognitive sensitivity that reflected ${ }_{221}$ the separation of these distributions, independent of any specific criterion for average per- ${ }^{222}$ formance. As shown in Figure 3D, completely overlapping distributions would fall along the ${ }_{223}$ equality line in an ROC plot, resulting in an Area Under the ROC curve (AUROC) of 0.5. ${ }^{224}$ In contrast, complete separation would yield an AUROC of 1. An advantage of this tech- ${ }^{225}$ nique over methods that rely on averaging (e.g., classification images) is that this method 226 is suitable for continuous performance distributions of any shape (e.g., skewed). There are ${ }^{227}$ two things worth noting about the interpretation of this metric. First, this is not the ROC ${ }_{228}$ method other researchers typically use to measure perceptual confidence (Barrett et al., 229 2013; Fleming and Lau, 2014). AUROC has, however, been used previously to explore the ${ }_{230}$ relationship between choice correctness and continuous confidence ratings as well as reaction 231 times (Faivre et al., 2018). Second, our AUROC measure has the following interpretation: ${ }^{232}$ if the experimenter was given the RMSE of two trials and was told one was rated "worse" ${ }_{233}$ and the other as "better", the AUROC would reflect the probability of correctly inferring 234 that the objectively better trial of the two was rated as "better" by the participant. 235

\subsection{Results}

Confirming the difficulty manipulation: We first examined whether the difficulty ma- ${ }^{237}$ nipulation affected objective tracking performance. Figure 4A shows the mean RMSE for ${ }_{238}$ each stimulus level for the two difficulty manipulations. Qualitatively, the difficulty levels ${ }_{239}$ appear matched for most participants: performance curves follow the equality line. To 240 
A

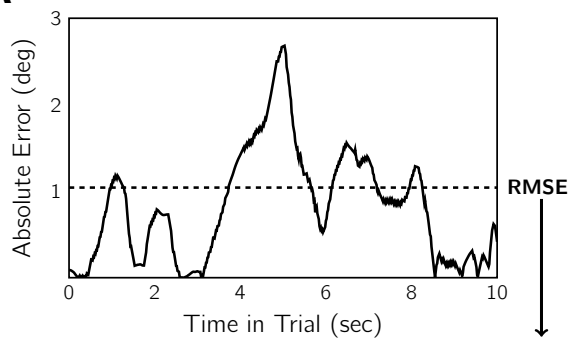

B

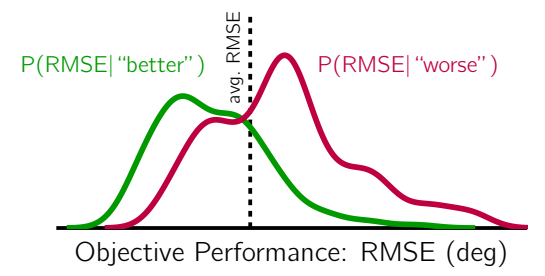

C

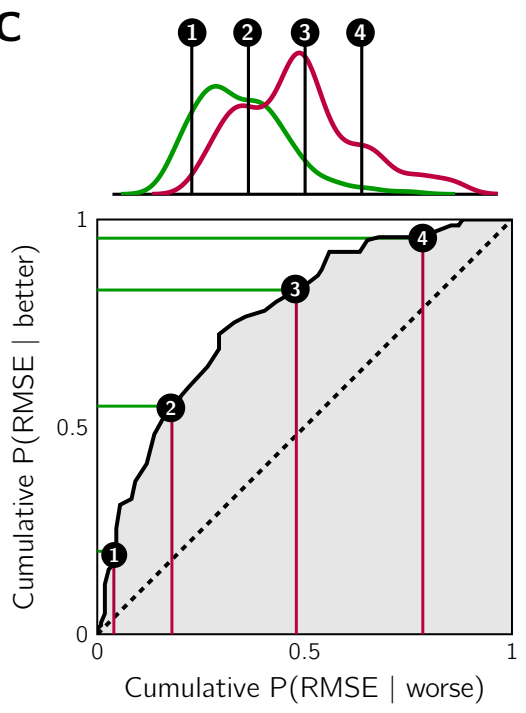

D

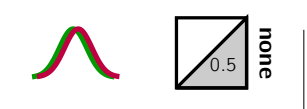

舟回

M国

Figure 3: A metacognitive sensitivity metric. A: Example of tracking error within a trial. Root-mean-squared-error (RMSE, dashed line) was the objective performance measure. B: Example participant's objective-error distributions, conditioned on sensorimotor confidence, for all trials in the variable cloud-size session. True average performance (dashed line) indicates the ideal criterion. Smaller RMSE tended to elicit "better" reports, and larger RMSE "worse". C: Metacognitive sensitivity was quantified by the separation of the conditional objective-error distributions with a non-parametric calculation of the Area Under the ROC (AUROC) using a quantile-quantile plot. At every point along the objectiveperformance axis, the cumulative probability of each conditional error distribution was contrasted. D: The area under the resulting curve is the AUROC statistic, with 0.5 indicating no meta-cognitive sensitivity and 1 indicating maximum sensitivity. The greater the separation of the conditional distributions, the more the objective tracking performance was predictive of sensorimotor confidence, and thus the higher the metacognitive sensitivity. 
check this result, we fit a generalised linear mixed-effects model (GLMM) to the RMSE ${ }_{241}$ values of each trial. The fixed effects in the model were difficulty manipulation (cloud-size ${ }_{242}$ or velocity-stability), stimulus difficulty (five levels), trial number, and an intercept term. ${ }^{243}$ The random effect was the participant. Trial number was included to test whether learn- ${ }^{244}$ ing occurred during the experiment. An analysis of deviance was performed using Type II ${ }_{245}$ Wald chi-square tests, revealing that only difficulty level had a significant effect on tracking 246 performance $\left(\chi^{2}=2042.85, p<.001\right)$. This confirms that the difficulty manipulations had 247 an impact on tracking performance. Also, the effect appears to be comparable across the 248 difficulty manipulations, as neither difficulty manipulation nor an interaction between dif- 249 ficulty manipulation and stimulus level had a significant effect $(p>0.05)$. As trial number 250 did not have a significant effect either $(p>0.05)$, training trials were likely sufficient for 251 performance to stabilise prior to the main task.

Overall metacognitive accuracy: Next, we examine metacognitive accuracy, which ${ }^{253}$ is the percentage of trials correctly judged as better or worse than average. Performance in 254 both sessions was significantly better than chance (cloud-size session: $66.3 \pm 1.8 \%$ correct; 255 velocity-stability session: $68.0 \pm 2.7 \%$ ). The accuracy results for each session are contrasted ${ }_{256}$ in Figure 4B. Two participants had significantly higher accuracy in the cloud-size session, 257 according to the $95 \%$ binomial error confidence intervals, and three participants were sig- ${ }^{258}$ nificantly more accurate in the velocity-stability session. Overall, evaluation of tracking 259 performance was similar in the two conditions. However, this accuracy metric may be 260 subject to response bias. Therefore, we examined meta-cognitive sensitivity. 261

Overall metacognitive sensitivity: A similar pattern of results was found for metacog- 262 nitive sensitivity. Metacognitive sensitivity (AUROC, see Methods) is contrasted between ${ }^{263}$ the sessions in Figure 4C and the individual ROC-style curves for cloud-size session and ${ }_{264}$ the velocity-stability session are shown in Figures 4D and 4E, respectively. All participants 265 displayed some degree of metacognitive sensitivity in both sessions: none of the ROC-style ${ }_{26}$ curves fell along the equality line. On average, the AUROC in the cloud-size session was ${ }^{267}$ 
A

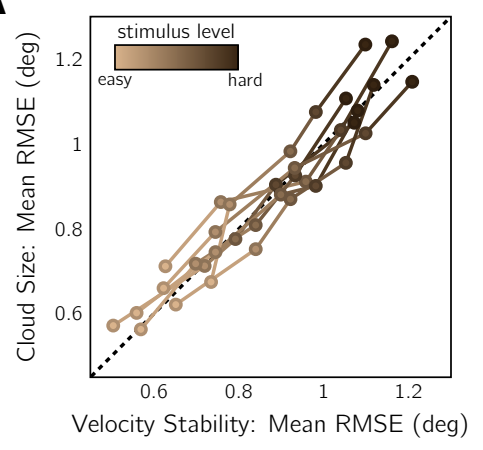

D

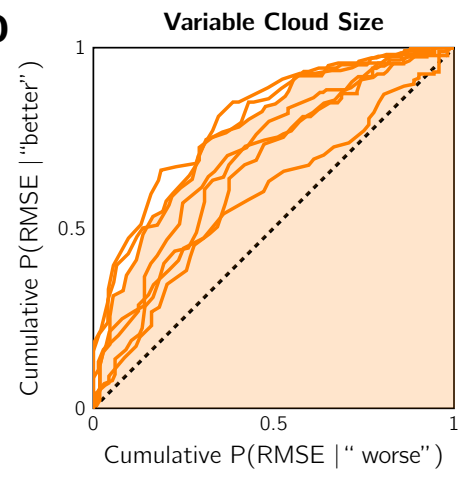

B

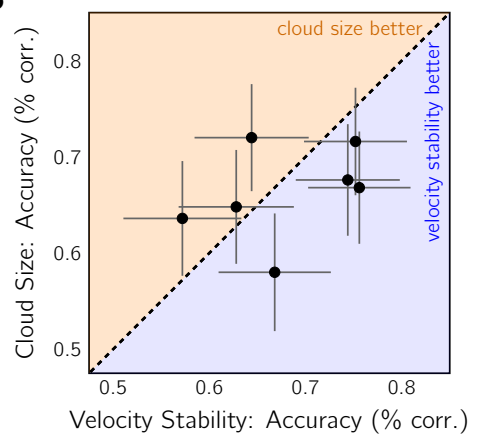

$E$

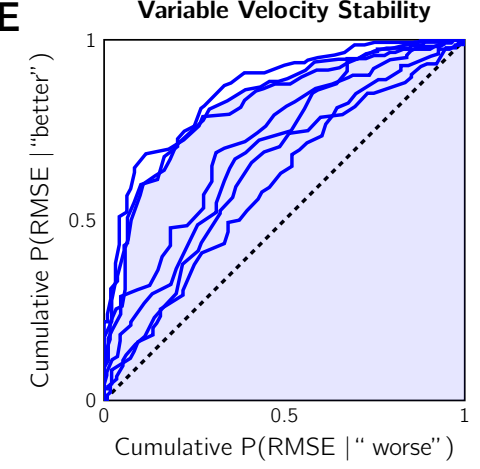

C

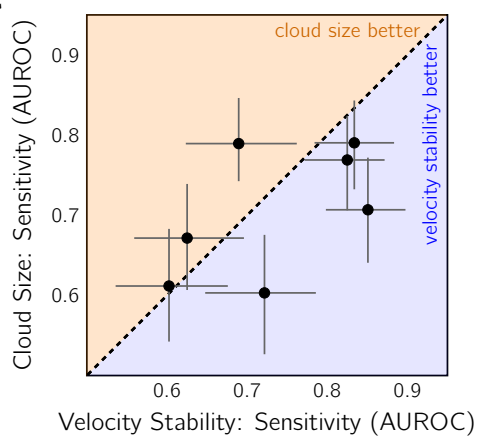

Figure 4: Comparable above-chance metacognitive sensitivity for cloud-size and velocitystability difficulty manipulations in Experiment $1(n=7)$. A: Effect of difficulty manipulation on tracking error. Mean RMSE contrasted for equivalent difficulty levels in the variable cloud-size session and the variable velocity-stability session. Colour: difficulty level. Curves: individual participants. Dashed line: equivalent difficulty. B: Comparison of metacognitive accuracy for the two difficulty-manipulation techniques, pooled across difficulty levels. Data points: individual subjects. Dashed line: equivalent accuracy. Error bars: 95\% binomial SE. Shaded regions indicate whether metacognitive accuracy was better for the cloud-size or velocity-stability session. C: Same as in (B) but comparing the sensitivity of the sensorimotor confidence judgement. Dashed line: equivalent sensitivity. Error bars: 95\% confidence intervals by non-parametric bootstrap. D: ROC-style curves for individual participants in the cloud-size session, pooled across difficulty levels. Shading: AUROC of example observer. Dashed line: the no-sensitivity lower bound. E: Same as (D) for the velocity-stability session. Shading corresponds to the same example observer. 
$0.71 \pm 0.03\left(\right.$ mean \pm SEM) and was $0.74 \pm 0.04$ for the velocity-stability session. At the group ${ }_{268}$ level, a Wilcoxon's Matched-Pairs Signed-Ranks Test revealed no significant difference be- ${ }^{269}$ tween AUROCs from the two sessions $(n=7, T=7, p>0.05)$. To examine the sensitivity 270 at the individual subject level, we performed a bootstrap procedure in which the AUROC ${ }_{271}$ was computed for each participant 1000 times, sampling from their trial set with replace- ${ }^{272}$ ment, allowing us to calculate $95 \%$ confidence intervals for our estimates (Figure 4C). Three ${ }_{273}$ participants were significantly more sensitive in the velocity-stability session, one was sig- ${ }^{274}$ nificantly more sensitive in the cloud-size session, and the other three showed no significant 275 difference between the two conditions. It is unlikely that these results are due to a learn- ${ }^{276}$ ing effect across sessions: 3 of the 4 significant results come from greater meta-cognitive ${ }_{277}$ accuracy in the first session completed. Another consideration is the amount of variability ${ }_{278}$ in performance for each individual and session. A highly variable participant may have ${ }^{279}$ a higher metacognitive sensitivity score because distinguishing better from worse perfor- ${ }_{280}$ mance is easier if a better trial differs more, on average, from a worse trial. Also, variance ${ }_{281}$ could have differed between the two difficulty manipulations, affecting within-participant ${ }_{282}$ comparisons of metacognitive sensitivity. To examine this we fit a GLMM of the AUROC ${ }_{283}$ with participant as the random effect, and fixed effects of RMSE variance (pooled across ${ }^{284}$ difficulty levels), difficulty manipulation, and an intercept term. We found no significant 285 effect of any of our predictors, indicating that performance variance likely did not play an ${ }^{286}$ important role in determining metacognitive sensitivity.

Temporal profile of metacognitive sensitivity: We conducted an analysis of metacog-288 nitive sensitivity for each $1 \mathrm{~s}$ time bin within the $10 \mathrm{~s}$ trial to examine the degree to which ${ }^{289}$ each second of tracking contributed to the final sensorimotor confidence judgement. An 290 AUROC of 0.5 indicates that error in that $1 \mathrm{~s}$ time bin has no predictive power for the ${ }_{291}$ metacognitive judgement; an AUROC of 1 indicates perfect predictive power. Figure 5A ${ }_{292}$ shows the results of this analysis. In both the cloud-size and the velocity-stability sessions ${ }_{293}$ there was a noticeable recency effect: error late in the trial was more predictive of sensori- ${ }^{294}$ 
motor confidence than error early in the trial. There was no discernible difference between 295 the two difficulty manipulations, except for the first few seconds where early error was more 296 predictive for the velocity-stability session.

For comparison, we also computed the temporal AUROCs, replacing the participant's 298 responses with simulated sensorimotor confidence judgements under two strategy extremes. 299 Figure 5B shows the AUROC time course for an ideal observer that had perfect knowledge 300 of performance (RMSE) and based the confidence judgment on whether the RMSE was 301 truly better or worse than average (i.e., weighted all time points equally). After the first 302 two seconds of tracking, the temporal AUROC is relatively level. Note that no time bin 303 was perfectly predictive of the confidence judgement, because the error within one second is 304 not equivalent to the total error across the entire trial. Figure 5C shows the AUROC time 305 course for an observer that perfectly uses uncertainty cues to judge the difficulty level of the 306 trial, and computes prospective confidence rather than basing the confidence judgment on 307 performance monitoring. Again, no single time bin should be particularly informative if one 308 is assessing a cue (e.g., dot-cloud size, velocity stability, etc) that does not disproportionately 309 occur at or affect performance for one particular portion of the trial. Confidence was coded 310 as "worse" for the two hardest difficulty levels, "better" for the two easiest, and flipping a 311 50-50 coin for the middle difficulty level. Again, both temporal profiles are flat after the 312 first 2 s. Neither perfect monitoring nor prospective confidence based on uncertainty cues 313 produced the recency effect in measured metacognitive behaviour. This result, however, is 314 not trivial due to the complex correlation structure of the error signal, which we investigated 315 next.

Weighing all time points equally is only an optimal strategy if all time bins are equally 317 predictive of trial-averaged performance. Error variability is one factor that can affect that: 318 periods of low error volatility have less impact on the predictive validity of a time bin 319 for overall RMSE. Thus, a recency effect might be an optimal strategy if there is higher 320 error volatility late in the trial. We found that error is overall lower and less variable 321 
A

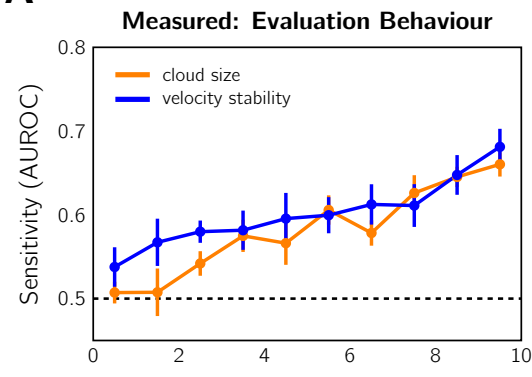

D

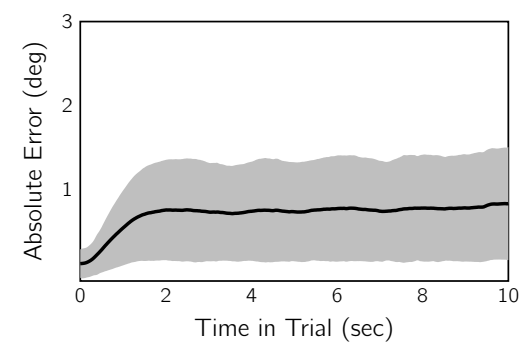

B

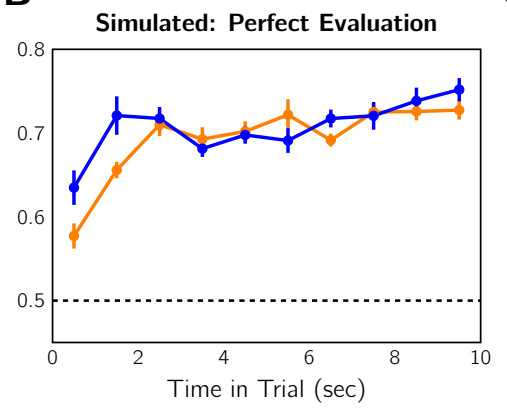

E

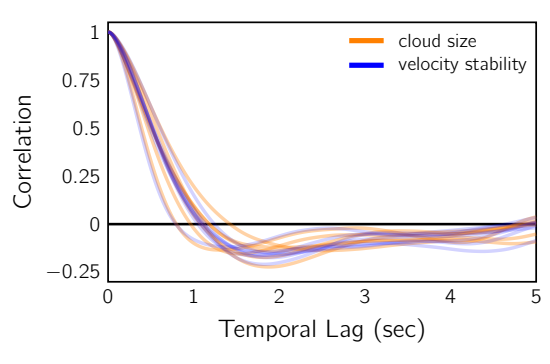

C
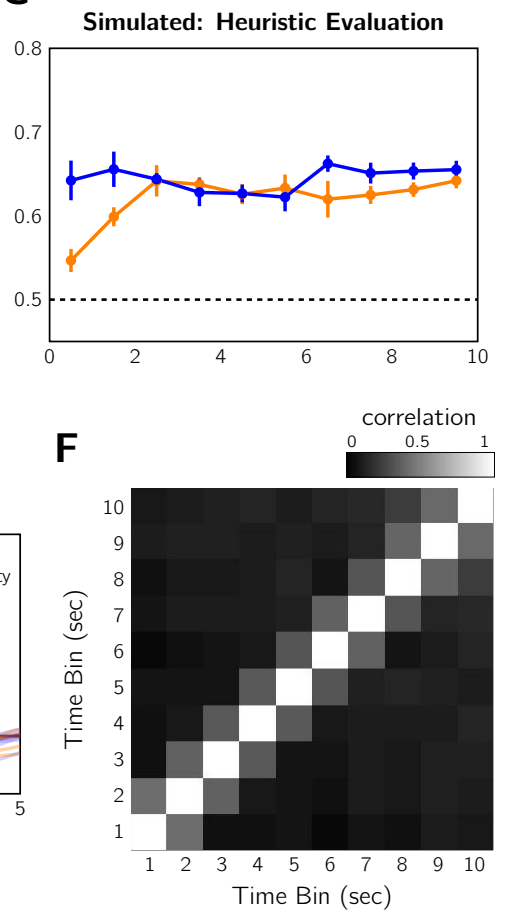

Figure 5: Performance weighting over time for sensorimotor confidence in Experiment $1(n=$ 7). A: AUROC analysis performed based on each 1-s time bin in the tracking period. Error bars: SEM across participants. Error later in the trial is more predictive of sensorimotor confidence as indicated by the higher AUROC. B: The same analysis as in (A) for an ideal observer that has perfect knowledge of the error and compares the RMSE to the average RMSE. C: Temporal analysis performed with simulated responses based on expected performance according to the heuristic of difficulty level (see text). D: Mean absolute error between target and cursor for all trials in both sessions shows error plateaus between 1-2 $\mathrm{s}$ and remains stable for the remainder of the trial. Grey region: \pm 1 SD. E: Auto-correlation of the tracking error signal for each subject and each session. F: Autocorrelation matrix of the $1 \mathrm{~s}$ binned RMSE. Data pooled over trials, conditions, and participants. The correlation between time-bins is relatively low after $1 \mathrm{~s}$. 
before $2 \mathrm{~s}$ (Figure 5D). This is because participants begin the trial by placing their cursor 322 at the centre of the screen, where the target is located. After this initial 2 s, however, 323 tracking error is constant in both mean and variance, indicating that all these time points 324 are equally informative on average about the final RMSE. Thus, error variance may explain 325 why metacognitive sensitivity was reduced for the initial $2 \mathrm{~s}$ for the measured and simulated 326 sensorimotor confidence, but it cannot explain the observed recency effect. Figure 5E shows 327 the auto-correlation of the signed error signal for each participant averaged across difficulty 328 levels. This graph reveals that error is correlated up to $\pm 1 \mathrm{~s}$, and is slightly anti-correlated 329 thereafter. Errors are necessarily related from moment to moment, due to the continuous 330 nature of tracking. To resolve a tracking error, one needs to make a corrective action to 331 compensate. The anti-correlation is likely a result of such corrective actions. Figure 5F 332 shows that this salient auto-correlation up to $\pm 1 \mathrm{~s}$ is also present between the RMSE of 333 neighbouring $1 \mathrm{~s}$ time bins. These results indicate that some of the predictive power of ${ }_{334}$ error in one time bin may be attributed to weighting of error in a neighbouring bin. Thus, 335 if we ask for what additional variance is accounted for, starting with last bin, the recency 336 effect would appear even stronger.

Other performance metrics: Our modelling thus far has been based on the error 338 between the location of the target and the cursor placement. However, this is not a realistic 339 model of how the participant perceives their error as they imperfectly infer target location 340 from the dot cloud, which is predominately affected by the external noise $\sigma_{\text {cloud }}$. To model ${ }_{341}$ this perceptual process (Figure 6A), we opted for a simple exponential filtering of the 342 centroid signal (i.e., the mid-point of the two dots presented each frame). The true centroid 343 position is a reasonable input, given that humans perform well at static centroid estimation 344 (McGowan et al., 1998; Juni et al., 2010). The smoothing aims to capture both the temporal 345 averaging in the visual system, which causes a cloud of 10 or so dots to be perceived, as well 346 as the averaging across time for strategic decision-making (Kleinman, 1969; Bonnen et al., 347 2015). The current estimate of target position, $\hat{x}_{t}$ is obtained by computing the weighted 348 
A

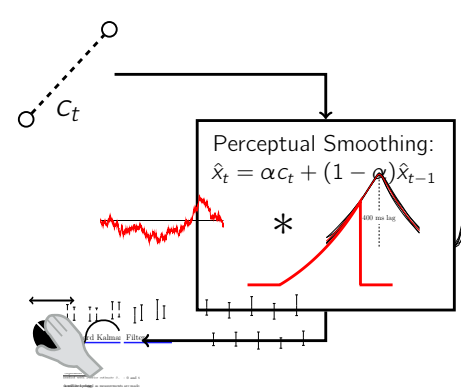

B

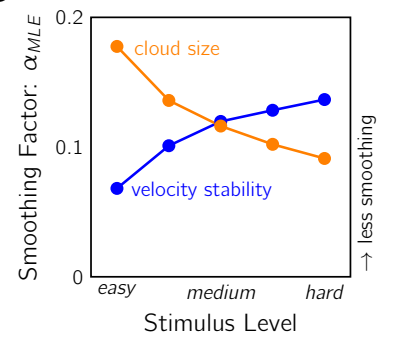

C

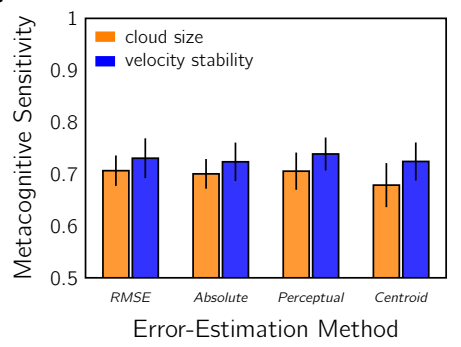

Figure 6: Comparing metacognitive sensitivity with different error-estimation methods. A: Diagram of the exponentially-smoothed perceptual model. Input: horizontal position of dot-cloud centroid, $c_{t}$ (i.e., dot midpoint on single frame). The perceptual system smooths the signal by convolving with an exponential to produce the target estimate $\hat{x}$. This is equivalent to the weighted sum of current input and previous estimate, $\hat{x}_{t-1}$, according to the smoothing parameter, $\alpha$. Output: perceived error determines the motor response. B: Setting of $\alpha$ that minimises the difference between true and perceived target location for each difficulty level and condition. C: Metacognitive sensitivity AUROC as measured under several error-estimation methods. RMSE: root-mean-squared-error between true target location and cursor. Absolute: mean absolute error between target and cursor. Perceptual: error according to the perceptual model in (A) with $\alpha$ values from (B). Centroid: RMSE calculated using dot-cloud centroid rather than true target location.

average at time, $t$, of the horizontal component of the current centroid, $c_{t}$, with the previous ${ }_{349}$ estimate, $\hat{x}_{t-1}$ :

$$
\hat{x}_{t}=\alpha c_{t}+(1-\alpha) \hat{x}_{t-1} \text {. }
$$

The smoothing parameter, $\alpha$, controls the steepness of the exponential. Larger $\alpha$ mean ${ }_{351}$ that current sensory evidence is weighted more than previous target estimates, and vice ${ }_{352}$ versa. The weighting is a trade-off that has to be balanced: averaging improves the amount 353 of information contributing to the estimate, but too much averaging into the past leads to 354 biased estimates.

We selected the value of $\alpha$ that minimised the sum of squared errors between true target 356 location and the model's estimate as a stand in for the observer's estimate of the current ${ }_{357}$ location of the target. This was calculated separately for each stimulus level and condition 358 (Figure 6B). As expected, there is less smoothing (larger $\alpha$ ) for the easy, small dot clouds 359 than the more difficult, large dot clouds (smaller $\alpha$ ). This is because accepting some history 360 
bias only makes sense when dealing with the noisier large dot clouds. The opposite pattern 361 is true for the velocity-stability condition. If velocity stability is high (easy), it is safer to 362 average further into the past to improve the estimate than if velocity stability is low (hard). 363 When the AUROC was calculated from the trial RMSE according to this perceptual model, 364 however, the results are relatively unchanged (Figure 6C). In fact, using the RMSE based 365 on the raw centroid signal also produced a similar AUROC estimate. We also examined 366 the mean absolute error, which is an alternate objective tracking metric to using RMSE, 367 and obtained the same result. The unchanging AUROC across these performance metrics 368 is likely due to the high correlation between all of these error measures. As compared to the 369 RMSE method, the correlations for the cloud-size condition are $r=0.98,0.93$, and 0.77 for 370 absolute error, perceptual error, and centroid error respectively. For the velocity-stability 371 condition, these are $r=0.98,0.94$, and 0.94. This is because all methods are measures 372 of the mean performance, which will change little with unbiased noise if given sufficient 373 samples (i.e., $10 \mathrm{~s}$ of tracking). Thus, we conclude that our AUROC statistic was a robust 374 measure and that the overlap in the confidence-conditioned distributions is unlikely due to 375 the selection of RMSE as the objective-performance metric.

Summary: Experiment 1 measured sensorimotor confidence for visuomotor tracking, 377 under both cloud-size and velocity-stability manipulations of difficulty, to address the three 378 goals of this study. A robust AUROC statistic, that quantified the ability of the confidence 379 judgements to distinguish objectively good from bad tracking, indicated that confidence 380 judgements were made with comparable above-chance metacognitive sensitivity for both 381 difficulty manipulations. Furthermore, a temporal analysis revealed a recency effect, where 382 tracking error later in the trial was found to disproportionately influence sensorimotor confi- ${ }_{383}$ dence. We propose that this is due to imperfect performance monitoring and not prospective 384 confidence based on heuristic cues to difficulty (i.e., cloud size, velocity stability). 


\section{Experiment 2}

The goal of Experiment 2 was to further investigate the recency effect. To this end, we re- 387 peated the task keeping the stimulus statistics fixed $\left(\sigma_{\text {cloud }}\right.$ and $\left.\sigma_{\text {walk }}\right)$ and instead varied the 388 duration of the stimulus presentation in an interleaved design. This made the time when the 389 sensorimotor-confidence judgement was required less predictable. Thus, participants would 390 be encouraged to sample error evidence for their confidence throughout the trial instead of 391 waiting until the final portion of the stimulus duration. If a response-expectation strategy 392 was the cause of the recency effect, we would expect to see flatter temporal AUROCs for this 393 mixed-duration design. Otherwise, if the recency effect is due to a processing limitation of 394 sensorimotor confidence, we would expect error in the last few seconds to largely determine 395 sensorimotor confidence regardless of the duration condition. Additionally, this experiment 396 allowed us to investigate sensorimotor confidence in the context of a fixed difficulty setting 397 that encourages participants to monitor their performance. This is because prospective 398 judgements of confidence, based on sensorimotor uncertainty, are uninformative when the 399 stimulus statistics are unchanging.

\subsection{Methods}

Participants: There were seven new participants in Experiment 2 (21-31 years old, one ${ }_{402}$ left-handed, four female). All participants had normal or corrected-to-normal vision and ${ }_{403}$ no self-reported motor abnormalities. Participants were naive to the purpose of the studies 404 except one author. Prior to the experiment, the task was described to the participants 405 and consent forms were collected. Participants were tested in accordance with the ethics 406 requirements of the Institutional Review Board at New York University.

Apparatus: All experiments were conducted on a Mac LCD monitor (Apple, Cuper- ${ }_{408}$ tino, CA; late 2013 version, 60 x 34 cm, 1920 x 1080 pixels, $60 \mathrm{~Hz}$ ), with participants 409 seated $57 \mathrm{~cm}$ from the monitor. Participants operated a Kensington M01215 wired optical ${ }_{410}$ 
mouse with their right hand when manually tracking the stimulus. Subjective performance ${ }_{411}$ evaluations were collected on a standard computer keyboard. Experiments were conducted 412 using custom-written code in MATLAB version R2014a (The MathWorks, Natick, MA), ${ }_{413}$ using Psychtoolbox version 3.0.12 (Brainard, 1997; Pelli, 1997; Kleiner et al., 2007). ${ }_{414}^{414}$

Task: Stimulus presentation duration was manipulated with an interleaved design and ${ }_{415}$ three levels $(6,10$, and $14 \mathrm{~s})$ while the stimulus statistics remained fixed at $\sigma_{\text {cloud }}=2 \operatorname{deg} \quad{ }^{416}$ and $\sigma_{\text {walk }}=0.15 \mathrm{deg} / \mathrm{s}$. Data were collected over three sessions, with each session composed ${ }_{417}$ of 15 training trials ( 5 per duration, randomised order) followed by 225 test trials (75 per ${ }_{418}$ duration, randomised order). Again, after each stimulus presentation, participants rated ${ }_{419}$ their subjective sense of their tracking performance as either "better" or "worse" than their 420 session average. As shown in Experiment 1, tracking before $2 \mathrm{~s}$ in this task has a different ${ }_{421}$ error profile, due to the target and cursor both starting at the same location from stationary. $\quad 422$ We opted to not count these initial $2 \mathrm{~s}$ of tracking in the final score so that trial duration ${ }_{423}$ could not serve as a difficulty manipulator in this experiment (e.g., a $6 \mathrm{~s}$ trial is more likely ${ }_{424}$ to have lower RMSE than a $14 \mathrm{~s}$ trial). In order to signal when the tracking contributed ${ }_{425}$ to the final score, the cursor was initially red (not contributing) and switched to green ${ }_{426}$ (contributing to the score) after 2 s. Furthermore, to ensure that all trials had the same ${ }_{427}$ stimulus statistics, all trajectories were sampled based on a 14 s stimulus and accepted or ${ }_{428}$ rejected before being temporally truncated if necessary. Tracking performance was scored ${ }_{429}$ and feedback given in the same manner as the previous experiment.

\subsection{Results}

In Experiment 2, we manipulated the duration of stimulus presentation with three inter- ${ }^{432}$ leaved conditions of 6,10 , or $14 \mathrm{~s}$. The consequence of duration on objective tracking ${ }^{433}$ performance was a small decrease in performance with longer durations (Figure 7A). The ${ }^{434}$ sensorimotor confidence judgements also showed slightly lower metacognitive accuracy (Fig- ${ }_{435}$ ure 7B) and sensitivity (Figure 7C) for longer durations. Overall, the average AUROC from ${ }_{436}$ 
A

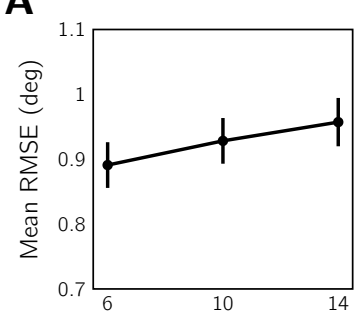

E

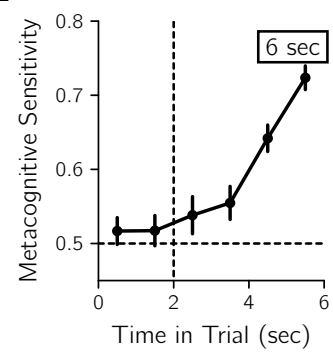

B

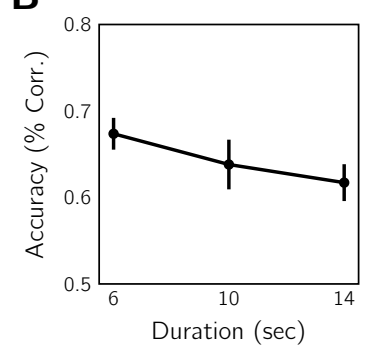

C
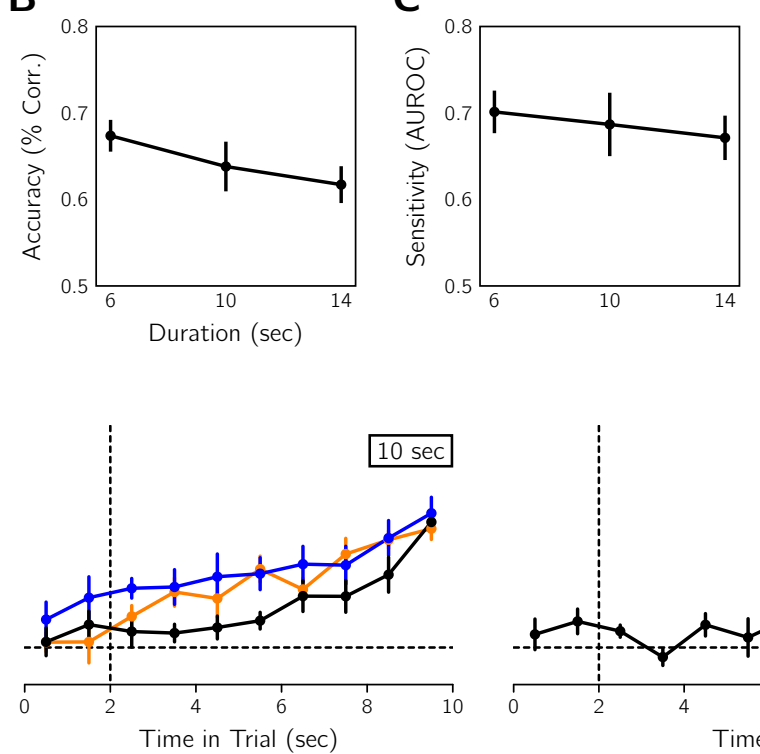

D

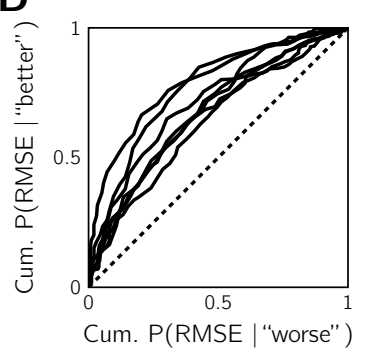

Figure 7: Recency effect replicated for variable stimulus-presentation duration in Experiment $2(n=7)$. A: Mean objective tracking performance for each duration condition averaged across observers. B: Sensorimotor-confidence accuracy for each duration condition. C: Metacognitive sensitivity for each duration condition. D: ROC-style curves for individual participants for AUROC pooled across durations. Dashed line: the no-sensitivity lower bound. Error before $2 \mathrm{~s}$ was excluded from the calculations in panels A-D. E: Temporal AUROCs calculated for $1 \mathrm{~s}$ time bins for each duration condition averaged across participants for Experiment 2 (black). For comparison, the results in Figure 5A are replotted (orange: cloud-size session; blue: velocity-stability session). Vertical dashed line at $2 \mathrm{~s}$ indicates the timing of cursor colour-change cue to begin evaluating tracking. Horizontal dashed line the no-sensitivity line. Error bars in all graphs are SEM. 
pooling data across durations was $0.68 \pm 0.04$ SEM (Figure 7D) and all participants had ${ }_{437}$ above-chance metacognitive sensitivity according to bootstrapped confidence intervals cal- ${ }^{438}$ culated as per the same procedure as Experiment 1. When split by session, the AUROCs ${ }_{439}$ were $0.68 \pm 0.04,0.68 \pm 0.03$, and $0.71 \pm 0.02$, suggesting that metacognitive performance ${ }_{440}$ was relatively unchanging across the sessions. Note that for these analyses we discarded ${ }_{441}^{4}$ the initial $2 \mathrm{~s}$ of tracking that the participants were instructed to ignore.

Figure 7E shows the temporal profile of metacognitive sensitivity for each duration as ${ }_{443}$ well as the results from Experiment 1. Participants were instructed to ignore tracking ${ }_{444}$ error occurring before $2 \mathrm{~s}$, when the cursor changed colour, for estimating sensorimotor ${ }_{445}$ confidence, and we observed low metacognitive sensitivity for these time points. Due to ${ }_{446}$ RMSE being partially correlated between adjacent time bins (Figure 5F), slightly elevated ${ }_{447}^{47}$ sensitivity for the time bin at $2 \mathrm{~s}$ does not necessarily indicate non-compliance with task ${ }_{448}$ instructions. For the remainder of the trial, later time points tend to have higher metacogni- ${ }_{449}$ tive sensitivity, consistent with the recency effect observed in Experiment 1. The steepness 450 of the temporal AUROC was also greater for shorter trial durations. This is to be expected ${ }_{451}$ as the contribution of a $1 \mathrm{~s}$ time bin to the final RMSE is greater when the trial is short. A ${ }_{452}$ recency effect is also consistent with the observed lower overall metacognitive performance ${ }_{453}$ for longer durations, because a smaller percentage of the total error signal contributes to ${ }_{454}$ sensorimotor confidence.

We observed a steeper recency effect for the $10 \mathrm{~s}$ condition than for the cloud-size or ${ }_{456}$ velocity-stability sessions in Experiment 1. Specifically, the sensitivity for the final time bin ${ }_{457}$ is comparable, but almost all other time bins have lower sensitivity than for the difficulty- ${ }_{458}$ manipulation results. However, it is unclear the extent to which the request that partici- ${ }^{459}$ pants ignore the first $2 \mathrm{~s}$ of tracking contributed to this difference, as this instruction was not 460 given in the previous experiment. We also attempted to compare the temporal AUROCs ${ }_{461}$ quantitatively with mixed success (see Supplementary Information). We found evidence for ${ }_{462}$ a stronger recency effect for Experiment 2 than Experiment 1. Furthermore, in our supple- ${ }_{463}$ 
mentary analyses, accounting for the recency effect and/or external noise via our perceptual ${ }_{464}^{4}$ model in Figure 5A gave little benefit when attempting to predict sensorimotor confidence ${ }_{465}$ for either experiment (at most $\sim 5 \%$ increase in predictive accuracy). However, we caution 466 against strong conclusions from these supplementary analyses as certain properties of the ${ }_{467}$ obtained data set were not ideal for these quantitative model fits.

468

In sum, We replicated the recency effect of Experiment 1 for all stimulus durations. ${ }^{469}$ Thus the final few seconds of tracking had the greatest influence on sensorimotor confi- 470 dence regardless of whether the participant knew when the stimulus would terminate. This ${ }^{471}$ suggests that response-expectation is unlikely to be the source of the recency effect. $\quad{ }_{472}$

\section{Discussion}

In two experiments, participants completed a visuomotor tracking task where trials were fol- ${ }_{474}$ lowed by a sensorimotor confidence judgement of "better" or "worse" than average tracking 475 performance. We calculated the degree to which these judgements predicted objective track- ${ }_{476}$ ing for manipulations of task difficulty (Experiment 1) and trial duration (Experiment 2), 477 with an AUROC metacognitive-sensitivity statistic that ranged from no sensitivity at 0.5478 and perfect sensitivity at 1 . In both experiments we found above-chance metacognitive 479 sensitivity and a temporal profile that suggested that error later in the trial contributed ${ }_{480}$ more to sensorimotor confidence.

\subsection{Performance monitoring}

One of our aims was to establish if humans would actively monitor their own performance ${ }_{483}$ to judge sensorimotor confidence. An alternate strategy would be to use cues to uncer- ${ }^{484}$ tainty (e.g., cloud size) to predict task difficulty and thus the likelihood of performing well. ${ }_{485}$ From our experiments, we found several indicators of performance monitoring. First, Ex- ${ }_{486}$ periment 1 manipulated task difficulty systematically with two methods, varying either the ${ }_{487}$ 
cloud-size parameter $\left(\sigma_{\text {cloud }}\right)$ or the velocity stability parameter $\left(\sigma_{\text {walk }}\right)$. The manipulation ${ }_{488}$ of $\sigma_{\text {cloud }}$ was very noticeable, with all participants reporting the stimulus manipulation in ${ }_{489}$ their debriefing interviews, whereas varying $\sigma_{\text {walk }}$ was more subtle and participants had 490 difficulty identifying the manipulation (supplementary media files are provided to demon- ${ }^{491}$ strate the difficulty manipulations). Thus, if the strategy was to rely exclusively on cues ${ }_{492}$ to uncertainty, and given that the manipulations had sizeable and comparable effects on 493 tracking performance, we would expect higher metacognitive sensitivity for the cloud-size ${ }_{494}$ session than the velocity-stability session. We did not find supporting evidence as there was 495 no significant difference in sensitivity between the sessions.

Stronger supporting evidence was found in Experiment 2, where task difficulty was 497 kept the same for all trials by fixing the stimulus statistics. In this scenario, there are no ${ }_{498}$ explicit uncertainty cues for the participant to use. Yet, metacognitive sensitivity was only ${ }_{499}$ slightly lower than was observed in Experiment 1 (AUROC of 0.68 in Experiment 2 versus 500 0.71 for cloud-size and 0.74 for velocity-stability in Experiment 1). However, variability 501 in tracking performance is not the same for fixed- and variable-difficulty designs; RMSE 502 differences are likely to be lower for a fixed-difficulty design, complicating the comparison. ${ }_{503}$ Furthermore, the difficulty manipulation in Experiment 1 may have permitted a mixed 504 strategy, combining performance monitoring and uncertainty heuristics. Thus, our results 505 from Experiment 2 supporting the performance-monitoring hypothesis are a better indicator 506 of how well performance monitoring captures true tracking performance than the results of 507 Experiment 1.

The best evidence for performance monitoring is the recency effect we observed in both 509 experiments. This is because it demonstrates that some moments in the trial influences sen- 510 sorimotor confidence more than others. Such a result is unlikely from the use of uncertainty 511 cues. For the cloud-size session, all time points equally signal the uncertainty, so there is 512 no reason that the final seconds should be privileged. Similarly, for the velocity-stability 513 session, the behaviour of the target would have to be observed for some period of time to 514 
assess velocity stability, but this could be done at any point during the trial. One possibility 515 is that participants were waiting until the end of the trial to make these assessments, but 516 the results of Experiment 2 argue against this, as the recency effect was still found when 517 stimulus-presentation duration was randomised. If instead participants were using some 518 other heuristic strategy (e.g., average velocity, amount of leftward motion, etc.), this would 519 also not produce a recency effect unless it predicted performance later in the trial but not 520 early performance. From an information-processing standpoint, performance monitoring is 521 likely to exhibit temporal sub-optimalities due to either leaky accumulation of the error 522 signal during tracking (Busemeyer and Townsend, 1993; Smith and Ratcliff, 2004) or the 523 temporal limitations of memory for retrospective judgements (Atkinson and Shiffrin, 1968; 524 Davelaar et al., 2005).

Before we examine the recency effect, we first comment on the possibility of a mixed 526 strategy of performance monitoring and uncertainty heuristics. Metacognitive judgements 527 based on a mixed strategy combining actual performance and cues to uncertainty have 528 been reported for sensorimotor confidence (Mole et al., 2018), motor-awareness confidence 529 (Charles et al., 2020), and perceptual confidence (De Gardelle and Mamassian, 2015; Spence 530 et al., 2015), with some exceptions (e.g., Barthelmé and Mamassian, 2010). Yet, it is 531 unclear if a mixed strategy was used in Experiment 1 of the present study. The obvi- ${ }_{532}$ ous/subtle distinction between the cloud-size and velocity-stability sessions that favours a 533 purely performance-monitoring strategy was not supported by a rigorous empirical test of 534 difficulty detectability. On the other hand, the recency effect appeared to be slightly weaker 535 for Experiment 1, suggesting another factor besides performance monitoring may have in- ${ }_{536}$ fluenced sensorimotor confidence, although our efforts to quantify this effect were hampered 537 by the nature of the error signals (see Supplementary information). An ideal test for use of 538 a mixed strategy would involve keeping performance constant by fixing the difficulty while 539 also varying likely uncertainty cues (e.g., titrating the mean and variability of the sensory 540 signal; De Gardelle and Mamassian, 2015; Spence et al., 2015). This is more difficult in sen- ${ }^{541}$ 
sorimotor tasks as motor variability will introduce noise into the error signal, hindering any 542 attempt to match performance. One way around this problem would be have participants 543 judge sensorimotor confidence for replays of previously completed tracking and artificially 544 adjust uncertainty cues. However, this would rely on metacognition acting similarly for 545 active tracking and passive viewing, which has only been confirmed for motor-awareness 546 confidence (Charles et al., 2020).

\subsection{The recency effect}

In the sensorimotor feedback process, incoming error signals inform upcoming action plans 549 and quickly become irrelevant (Todorov, 2004; Bonnen et al., 2015). In contrast, the goal 550 of performance monitoring for sensorimotor confidence is to accumulate error signals across 551 time, much like the accumulation of sensory evidence for perceptual decisions with a fixed 552 viewing time. In fact, in the accumulation-of-evidence framework, considerable effort has 553 been made to incorporate a recency bias termed "leaky accumulation" (Busemeyer and 554 Townsend, 1993; Usher and McClelland, 2001; Brunton et al., 2013; Matsumori et al., 2018). 555 The main arguments for including a temporal-decay component is to account for memory 556 limitations of the observer (e.g., from neural limits of recurrent excitation) or intentional 557 forgetting for adaptation in volatile environments (Usher and McClelland, 2001; Nassar ${ }_{558}$ et al., 2010; Norton et al., 2019). For our task, memory constraints are a more likely 559 explanation of the recency effect than intentional forgetting, because we have long trials of 560 6-14 s with no changes of stimulus statistics during a trial. One contributor to the error 561 signal we have no control over, however, is the participant's motivation to do the task. 562 Even though tracking performance was constant when averaged across trials, fluctuations 563 in motivation during a trial could lead to fluctuations in motor performance that do cause 564 volatility in the error signal. Thus, alternating between bouts of good and poor performance 565 could bias the participant to be more forgetful.

Previous efforts to characterise the time course of a metacognitive judgement have been 567 
limited to the perceptual domain. Using the reverse-correlation technique, Zylberberg et al. 568 (2012) measured the temporal weighting function for confidence in two perceptual tasks and 569 found a primacy effect: the initial hundreds of milliseconds of stimulus presentation had the 570 greatest influence on perceptual confidence. Their finding and associated modelling suggests 571 evidence accumulation for the metacognitive judgement stops once an internal bound for 572 decision commitment has been reached. Our results suggest that sensorimotor confidence 573 does not follow the same accumulation-to-bound structure, otherwise early error would have 574 been more predictive of confidence than late error. One reason we may not have found a 575 primacy effect is that the participant interacts with the stimulus to produce the errors that 576 determine performance, allowing them a sense of agency that they can change or modify 577 performance. As a result, there is no reason to settle on a confidence judgement based 578 on initial performance. A contradictory finding to Zylberberg et al. (2012) is that sensory 579 evidence late in the trial, during the period between the sensory decision and the metacog- 580 nitive decision, can influence perceptual confidence in what is termed post-accumulation of 581 evidence (Pleskac and Busemeyer, 2010), but this finding is hard to apply to our visuomotor 582 task. Evaluating tracking is different from a single perceptual decision, because tracking is 583 a series of motor-planning decisions (Wolpert and Landy, 2012). The error signal used to 584 plan the next tracking movement is also the feedback of the error from the last moment 585 of tracking. Additionally, subsequent estimates of target location could theoretically pro- 586 vide additional information about previous locations of the target. Identifying the source 587 of the error signal for sensorimotor confidence, either by computational modelling or brain 588 imaging, would help clarify the nature of the accumulation process. 589

So far we have considered an online computation of metacognition in parallel with sen- 590 sorimotor decision making. Another alternative is that the evaluation of performance is 591 computed retrospectively. Baranski and Petrusic (1998) showed that reaction times for 592 confidence responses differed for speeded and unspeeded perceptual decisions, leading to 593 the conclusion that perceptual confidence is computed online unless time pressure forces 594 
it to be evaluated retrospectively. It is reasonable to assume that the continual demand 595 of cursor adjustment to track an unpredictable stimulus is taxing, leaving participants no 596 choice but to introspect on their performance upon termination of the trial. If this were the ${ }_{597}$ case, we would likely see temporal biases consistent with memory retrieval. In the memory 598 literature, there has been extensive evidence of both primacy and recency effects, which 599 are thought to be associated with long-term and short-term memory processes respectively 600 (Atkinson and Shiffrin, 1968; Innocenti et al., 2013). Thus, the observed recency effect in 601 our experiment could be the result of short-term memory limitations constraining the time 602 constant. Another reason observers may delay performance evaluation until after the trial ${ }_{603}$ is because tracking is typically a goal-directed behaviour, which can be evaluated by its 604 success (e.g., catching the prey after a chase, hitting the target in a first-person shooter ${ }_{605}$ game, or correctly intercepting a hand in a handshake). Still, one may want to introspect ${ }_{606}$ about performance while tracking to decide whether the tracking was in vain. We did not ${ }_{607}$ incentivise participants to adopt a particular strategy in the task, so they may have treated ${ }_{608}$ error towards the end of the trial as their success in "catching" the target.

\subsection{Metacognitive efficiency}

We quantified metacognitive sensitivity for sensorimotor tracking with an AUROC metric ${ }_{611}$ that reflected the separation of the objective-performance distributions conditioned on sen- ${ }_{612}$ sorimotor confidence. This approach superficially shares some similarities with the metacog- 613 nitive metric meta- $d^{\prime}$ in perceptual confidence. For meta- $d^{\prime}$, an ROC curve, relating the ${ }_{614}$ probability of a confidence rating conditioned on whether the observer was correct vs. incor- ${ }_{615}$ rect, is computed as part of the analysis to obtain a bias-free sensitivity metric that reflects ${ }_{616}$ the observer's ability to distinguish between correct and incorrect perceptual responses ${ }_{617}$ (Fleming and Lau, 2014; Mamassian, 2016). However, the area under this ROC curve ${ }_{618}$ (AUROC) has little meaning, as it is highly dependent on the sensitivity of the primary ${ }_{619}$ perceptual judgement (Galvin et al., 2003). Instead, the appropriate comparison is between 620 
the perceptual sensitivity, $d^{\prime}$, and the metacognitive sensitivity, meta- $d^{\prime}$. Typically, a ratio ${ }_{621}$ of these sensitivities is computed, with a value of 1 being considered ideal metacognitive ${ }_{622}$ efficiency (i.e., the best the observer can do given the identical sensory evidence available ${ }_{623}$ for the metacognitive judgement as the perceptual judgement). Empirically, ratios less ${ }_{624}$ than 1 are most often observed, indicating less efficient, more noisy decision-making at the ${ }_{625}$ metacognitive level (Maniscalco and Lau, 2012, 2016).

In contrast, the purpose of our AUROC metric is not to quantify how well the sensory ${ }_{627}$ information is used for the sensorimotor control versus sensorimotor confidence. Instead we ${ }_{628}$ used it as a non-parametric way of quantifying how sensitive an observer is to their true ${ }_{629}$ performance. The metric ranges from no sensitivity (i.e., chance performance) at 0.5 to 630 perfect classification performance at 1 . As with perceptual confidence, we do expect that the ${ }_{631}$ AUROC will depend to some degree on the variance in the performance of the primary task 632 (e.g., tracking). For example, if there is little variance, then it should be difficult to identify ${ }_{633}$ well executed from poorly executed trials, whereas a large variance means performance could ${ }_{634}$ be more easily categorised. A second use of the AUROC metric was to quantify the degree ${ }_{635}$ to which a model of metacognitive behaviour could predict sensorimotor confidence (see ${ }_{636}$ Supplementary Information). By replacing the objective-performance axis with an internal ${ }_{637}$ decision-variable axis according to a model, a model's explanatory power can be measured ${ }_{638}$ on a scale from none at 0.5 to perfect at 1 . While we were unsuccessful at improving ${ }_{639}$ performance more than $5 \%$ in any of our experiments, which we did by accounting for ${ }_{640}$ both the recency effect and the effect of external sensory noise instead of simply computing ${ }_{641}$ RMSE using the true target location, the method of analysis nicely complemented our goal ${ }_{642}$ of quantifying how well sensorimotor confidence reflected objective performance.

We instead examined metacognitive efficiency by determining what error information 644 contributed to sensorimotor confidence. The recency effect we observed constitutes an in- ${ }_{645}$ efficiency in that not all information used for the primary sensorimotor decision-making 646 was used for the metacognitive judgement as was instructed. Based on the similarity in 647 
shape of the recency effect for the duration conditions of Experiment 2, we can conclude ${ }_{648}$ that efficiency is inversely proportional to the duration of tracking. However, given long, ${ }_{649}$ multi-action sequences, it is not that surprising to find that some part of the perceptual in- ${ }_{650}$ formation about error is lost. Some amount of forgetting is likely advantageous in real-world 651 scenarios. For future metacognitive studies of action, it would be informative to examine es- ${ }_{652}$ timates of sensorimotor confidence during action and how sensorimotor confidence interacts 653 with goal planning, explicit learning, and expertise. For example, it would be worthwhile ${ }_{654}$ to investigate how sensorimotor confidence relates to cognitive control functions such as 655 switching or abandoning motor tasks (Alexander and Brown, 2010), or how athletes and 656 novices judge sensorimotor confidence (MacIntyre et al., 2014).

\subsection{Conclusion}

In sum, we found considerable evidence that humans are able to compute sensorimotor ${ }_{659}$ confidence, that is, they are able to monitor their motor performance in relationship to a 660 goal. However, they do so inefficiently, in particular because of the recency effect that we ${ }_{661}$ revealed, disproportionately weighting the tracking error at the end of the trial to judge ${ }_{662}$ whether their performance was better than average. We replicated this effect with unpre- ${ }_{663}$ dictable stimulus-presentation durations to confirm that it was not the result of a response- ${ }_{664}^{6}$ preparation strategy. In our analyses, we have introduced the AUROC statistic, which 665 we found useful for two purposes. First, it allowed us to quantify the relationship be- ${ }_{66}$ tween sensorimotor confidence and objective tracking performance, and second, it provided ${ }_{667}$ a model-fit metric for elaborated decision models. Our results, obtained from a relatively ${ }_{668}$ simple goal of visuo-motor tracking, raise many questions for future studies on sensorimotor ${ }_{669}$ confidence. For example, is the recency effect a general phenomenon for sensorimotor con- 670 fidence? And, does it result from online evidence accumulation of error or a retrospective 671 memory retrieval upon termination of movement? What factors determine the strength 672 of the recency effect for sensorimotor confidence (i.e., attention, sensorimotor goals, etc.)? 673 
Further work will help provide a clearer link between models of sensorimotor behaviour and ${ }_{674}$ models of motor metacognition.

\section{Acknowledgements}

We thank Eero Simoncelli for fruitful discussions that contributed to this project. This work

677

was supported by NIH Grant EY08266 and National Science Foundation Collaborative

678

Research in Computational Neuroscience Grant 1420262, as well as French ANR grant 679

ANR-18-CE28-0015-01"VICONTE".

\section{Author note}

This research was presented at the 2017 Vision Sciences Society meeting at St. Pete Beach, ${ }_{682}$ FL and the 2017 Conference on Cognitive Computational Neuroscience in New York, NY. ${ }_{683}$ Data from this study can be found at https://osf.io/enxdt/. Individual author con- ${ }^{6} 64$ tributions in the CRediT taxonomy style are as follows (black: major contribution, gray: 685 minor contribution, see https://www.casrai.org/credit.html for more details):

\begin{tabular}{|c|c|c|c|}
\hline & SML & PM & MSL \\
\hline Conceptualization & \multicolumn{2}{|l|}{} \\
\cline { 1 - 1 } Data curation & \multirow{2}{|l|}{} & \\
\hline Formal analysis & & & \\
\hline Funding acquisition & & & \\
\hline Investigation & & & \\
\hline Methodology & & \\
\hline Project administration & & & \\
\hline Resources & & & \\
\hline Software & & & \\
\hline Supervision & & & \\
\hline Validation & & & \\
\hline Visualization & & \\
\hline Writing - original draft & & \\
\hline Writing - review \& editing & & \\
\hline
\end{tabular}




\section{References}

Adler, W. T. and Ma, W. J. (2018). Comparing Bayesian and non-Bayesian accounts of ${ }_{688}$ human confidence reports. PLoS Computational Biology, 14(11):e1006572.

Alexander, W. H. and Brown, J. W. (2010). Computational models of performance moni- ${ }_{690}$ toring and cognitive control. Topics in Cognitive Science, 2(4):658-677.

Atkinson, R. C. and Shiffrin, R. M. (1968). Human Memory: A Proposed System and Its 692 Control Processes. Psychology of Learning and Motivation, 2:89-195.

Augustyn, J. S. and Rosenbaum, D. A. (2005). Metacognitive control of action: Preparation 694 for aiming reflects knowledge of Fitts's law. Psychonomic Bulletin and Review, 12(5):911- ${ }^{695}$ 916.

Baranski, J. V. and Petrusic, W. M. (1998). Probing the Locus of Confidence Judgments: ${ }_{697}$ Experiments on the Time to Determine Confidence. Journal of Experimental Psychology: ${ }_{698}$ Human Perception and Performance, 24(3):929-945.

Barrett, A. B., Dienes, Z., and Seth, A. K. (2013). Measures of metacognition on signal- 700 detection theoretic models. Psychological Methods, 18(4):535-552.

Barthelmé, S. and Mamassian, P. (2010). Flexible mechanisms underlie the evaluation of 702 visual confidence. Proceedings of the National Academy of Sciences, 107(48):20834-20839. 703

Bègue, I., Blakemore, R., Klug, J., Cojan, Y., Galli, S., Berney, A., Aybek, S., and Vuilleu- ${ }^{704}$ mier, P. (2018). Metacognition of visuomotor decisions in conversion disorder. Neuropsy- 705 chologia, 114:251-265.

Blakemore, S.-J. and Frith, C. (2003). Self-awareness and action. Current Opinion in 707 Neurobiology, 13(2):219-224.

Blakemore, S. J., Wolpert, D. M., and Frith, C. D. (2002). Abnormalities in the awareness 709 of action. Trends in Cognitive Sciences, 6(2):237-242.

Bonnen, K., Yates, J., Burge, J., Pillow, J., and Cormack, L. K. (2015). Continuous 711 psychophysics: Target-tracking to measure visual sensitivity. Journal of Vision, 15(3):14. 712

Botvinick, M. M., Braver, T. S., Barch, D. M., Carter, C. S., and Cohen, J. D. (2001). 713 Conflict monitoring and cognitive control. Psychological Review, 108(3):624-652. 714

Brainard, D. H. (1997). The Psychophysics Toolbox. Spatial Vision, 10(4):433-436. 715 
Brunton, B. W., Botvinick, M. M., and Brody, C. D. (2013). Rats and humans can optimally 716 accumulate evidence for decision-making. Science, 340(6128):95-98. 717

Busemeyer, J. R. and Townsend, J. T. (1993). Decision field theory: A dynamic- 718 cognitive approach to decision making in an uncertain environment. Psychological Review, 719 100(3):432-459.

720

Charles, L., Chardin, C., and Haggard, P. (2020). Evidence for metacognitive bias in 721 perception of voluntary action. Cognition, 194:104041. 722

Davelaar, E. J., Goshen-Gottstein, Y., Ashkenazi, A., Haarmann, H. J., and Usher, M. ${ }^{723}$ (2005). The demise of short-term memory revisited: Empirical and computational inves- 724 tigations of recency effects. Psychological Review, 112(1):3-42.

725

De Gardelle, V. and Mamassian, P. (2015). Weighting mean and variability during confi- 726 dence judgments. PLoS ONE, 10(3):e0120870.

Dotan, D., Meyniel, F., and Dehaene, S. (2018). On-line confidence monitoring during ${ }_{728}$ decision making. Cognition, 171:112-121.

729

Faivre, N., Filevich, E., Solovey, G., Kühn, S., and Blanke, O. (2018). Behavioral, modeling, 730 and electrophysiological evidence for supramodality in human metacognition. Journal of 731 Neuroscience, 38(2):263-277.

732

Fleming, S. M. and Daw, N. D. (2017). Self-evaluation of decision-making: A general ${ }_{733}$ Bayesian framework for metacognitive computation. Psychological Review, 124(1):91- ${ }^{734}$ 114.

Fleming, S. M. and Dolan, R. J. (2012). The neural basis of metacognitive ability. Philo- 736 sophical Transactions of the Royal Society B: Biological Sciences, 367(1594):1338-1349. ${ }_{737}$

Fleming, S. M. and Lau, H. C. (2014). How to measure metacognition. Frontiers in Human 738 Neuroscience, 8:443. 739

Fourneret, P. and Jeannerod, M. (1998). Limited conscious monitoring of motor perfor- 740 mance in normal subjects. Neuropsychologia, 36(11):1133-1140. 741

Galvin, S. J., Podd, J. V., Drga, V., and Whitmore, J. (2003). Type 2 tasks in the theory of ${ }_{742}$ signal detectability: Discrimination between correct and incorrect decisions. Psychonomic ${ }_{743}$ Bullentin \& Review, 10(4):843-876. 
Innocenti, I., Cappa, S. F., Feurra, M., Giovannelli, F., Santarnecchi, E., Bianco, G., Cin- 745 cotta, M., and Rossi, S. (2013). TMS interference with primacy and recency mechanisms 746 reveals bimodal episodic encoding in the human brain. Journal of Cognitive Neuroscience, 747 25(1):109-116.

Juni, M. Z., Singh, M., and Maloney, L. T. (2010). Robust visual estimation as source 749 separation. Journal of Vision, 10(14):2.

Kiani, R., Corthell, L., and Shadlen, M. N. (2014). Choice certainty is informed by both 751 evidence and decision time. Neuron, 84(6):1329-1342.

Kleiner, M., Brainard, D., Pelli, D., Ingling, A., Murray, R., and Broussard, C. (2007). ${ }^{753}$ What's new in psychtoolbox-3. Perception, 36(14):1-16.

Kleinman, D. (1969). Optimal control of linear systems with time-delay and observation 755 noise. IEEE Transactions on Automatic Control, 14(5):524-527.

MacIntyre, T. E., Igou, E. R., Campbell, M. J., Moran, A. P., and Matthews, J. (2014). 757 Metacognition and action: A new pathway to understanding social and cognitive aspects 758 of expertise in sport. Frontiers in Psychology, 5:1155.

Mamassian, P. (2016). Visual Confidence. Annual Review of Vision Science, 2(1):459-481. 760

Maniscalco, B. and Lau, H. (2016). The signal processing architecture underlying subjective 761 reports of sensory awareness. Neuroscience of Consciousness, 2016(1):1-17. 762

Maniscalco, B. and Lau, H. C. (2012). A signal detection theoretic approach for estimating 763 metacognitive sensitivity from confidence ratings. Consciousness and Cognition, 21:422- 764 430 .

Matsumori, K., Koike, Y., and Matsumoto, K. (2018). A biased Bayesian inference for 766 decision-making and cognitive control. Frontiers in Neuroscience, 12:734. 767

McGowan, J. W., Kowler, E., Sharma, A., and Chubb, C. (1998). Saccadic localization of 768 random dot targets. Vision Research, 38(6):895-909.

Mole, C. D., Jersakova, R., Kountouriotis, G. K., Moulin, C. J. A., and Wilkie, R. M. 770 (2018). Metacognitive judgements of perceptual-motor steering performance. Quarterly 771 Journal of Experimental Psychology, 71(10):2223-2234. 
Nassar, M. R., Wilson, R. C., Heasly, B., and Gold, J. I. (2010). An Approximately Bayesian 773 Delta-Rule Model Explains the Dynamics of Belief Updating in a Changing Environment. 774 Journal of Neuroscience, 30(37):12366-12378.

Norman, D. A. and Shallice, T. (1986). Attention to action. In Consciousness and self- 776 regulation, pages $1-18$. Springer.

Norton, E. H., Acerbi, L., Ma, W. J., and Landy, M. S. (2019). Human online adaptation 778 to changes in prior probability. PLoS Computational Biology, 15(7):e1006681.

Patel, D., Fleming, S. M., and Kilner, J. M. (2012). Inferring subjective states through 780 the observation of actions. Proceedings of the Royal Society B: Biological Sciences, 781 279(1748):4853-4860.

Pelli, D. G. (1997). The Video Toolbox software for visual psychophysics: Transforming 783 numbers into movies. Spatial Vision, 10:437-442.

Pleskac, T. J. and Busemeyer, J. R. (2010). Two-stage dynamic signal detection: A theory 785 of choice, decision time, and confidence. Psychological Review, 117(3):864-901.

Pouget, A., Drugowitsch, J., and Kepecs, A. (2016). Confidence and certainty: distinct 787 probabilistic quantities for different goals. Nature Neuroscience, 19(3):366-374.

Resulaj, A., Kiani, R., Wolpert, D. M., and Shadlen, M. N. (2009). Changes of mind in 789 decision-making. Nature, 461(7261):263-266.

Sinanaj, I., Cojan, Y., and Vuilleumier, P. (2015). Inter-individual variability in metacogni- 791 tive ability for visuomotor performance and underlying brain structures. Consciousness 792 and Cognition, 36:327-337.

Smith, P. L. and Ratcliff, R. (2004). Psychology and neurobiology of simple decisions. 794 Trends in Neurosciences, 27(3):161-168.

Spence, M. L., Dux, P. E., and Arnold, D. H. (2015). Computations underlying confi- 796 dence in visual perception. Journal of Experimental Psychology: Human Perception and 797 Performance, 42(5):671-682.

Stevenson, I. H., Fernandes, H. L., Vilares, I., Wei, K., and Körding, K. P. (2009). Bayesian 799 integration and non-linear feedback control in a full-body motor task. PLoS Computa- 800 tional Biology, 5(12):e1000629. 
Todorov, E. (2004). Optimality principles in sensorimotor control. Nature Neuroscience, 802 $7(9): 907-915$.

Trommershäuser, J., Maloney, L. T., and Landy, M. S. (2008). Decision making, movement 804 planning and statistical decision theory. Trends in Cognitive Sciences, 12(8):291-297. ${ }_{805}$

Usher, M. and McClelland, J. L. (2001). The time course of perceptual choice: The leaky, 806 competing accumulator model. Psychological Review, 108(3):550-592.

Wolpert, D. M., Ghahramani, Z., and Jordan, M. I. (1995). An internal model for sensori- 808 motor integration. Science, 269(5232):1880-1882.

Wolpert, D. M. and Landy, M. S. (2012). Motor control is decision-making. Current Opinion 810 in Neurobiology, 22(6):996-1003.

Yeung, N. and Summerfield, C. (2012). Metacognition in human decision-making: Confi- ${ }_{812}$ dence and error monitoring. Philosophical Transactions of the Royal Society B: Biological ${ }^{813}$ Sciences, 367(1594):1310-1321.

Zhang, H., Daw, N. D., and Maloney, L. T. (2013). Testing Whether Humans Have an 815 Accurate Model of Their Own Motor Uncertainty in a Speeded Reaching Task. PLoS ${ }_{816}$ Computational Biology, 9(5):e1003080.

Zylberberg, A., Barttfeld, P., and Sigman, M. (2012). The construction of confidence in a 818 perceptual decision. Frontiers in Integrative Neuroscience, 6:79. 